UUITP-04/07

HIP-2007-12/TH

YITP-SB-07-09

\title{
A potential for Generalized Kähler Geometry
}

\author{
Ulf Lindström ${ }^{a, b}$, Martin Roček ${ }^{c}$, Rikard von Unge $^{d}$, and Maxim Zabzine ${ }^{a}$ \\ ${ }^{a}$ Department of Theoretical Physics Uppsala University, \\ Box 803, SE-751 08 Uppsala, Sweden \\ ${ }^{b}$ HIP-Helsinki Institute of Physics, University of Helsinki, \\ P.O. Box 64 FIN-00014 Suomi-Finland \\ ${ }^{c}$ C.N.Yang Institute for Theoretical Physics, Stony Brook University, \\ Stony Brook, NY 11794-3840,USA \\ ${ }^{d}$ Institute for Theoretical Physics, Masaryk University, \\ 61137 Brno, Czech Republic
}

\begin{abstract}
We show that, locally, all geometric objects of Generalized Kähler Geometry can be derived from a function $K$, the "generalized Kähler potential". The metric $g$ and two-form $B$ are determined as nonlinear functions of second derivatives of $K$. These nonlinearities are shown to arise via a quotient construction from an auxiliary local product (ALP) space.
\end{abstract}




\section{Introduction}

Generalized Kähler Geometry [1] is a special and particularly interesting example of Generalized Geometry [2, 1]. It succinctly encodes the bihermitean geometry of Gates, Hull and Roček [3] in terms of a pair of commuting generalized complex structures $\left(\mathcal{J}_{1}, \mathcal{J}_{2}\right)$. Here we use the equivalent bihermitean data to give a complete (local) description of Generalized Kähler Geometry. We show that there exists a function $K$ with the interpretation of a generating function for symplectomorphisms between two sets of coordinates in terms of which all geometric quantities may be described. In particular, the combination $E=\frac{1}{2}(g+B)$ of the metric and antisymmetric $B$-field is given as a nonlinear expression in terms of second derivatives of $K$. When further analyzed, this expression has the structure of a quotient from some higher dimensional space. We find this auxiliary space, which we call an ALP space, and display the quotient structure. Finding a Generalized Kähler Geometry is thus reduced to a linear construction in terms of the corresponding $K$ on the ALP.

The bihermitean geometry was first derived as the target space geometry of $\mathcal{N}=(2,2)$ supersymmetric nonlinear sigma models [3]. Correspondingly, all our constructions have a sigma model realization, and many of the proofs were derived in that setting. In particular, the "generalized Kähler potential" $K$ has an interpretation as the superspace sigma model Lagrangian1. Detailed descriptions of this approach may be found in the two articles on which the presentation is based [5, 6].

\section{Generalized Complex Geometry (GCG)}

This section contains a brief recapitulation of the salient features of GCG. The references for the mathematical aspects are chiefly [2, 1, while a presentation more accessible to physicists (containing, e.g., coordinate and matrix descriptions) may be found in [7, 8].

A generalized almost complex structure is an algebraic structure on the sum of the tangent and cotangent bundles of a manifold $\mathcal{M}$ defined via

$$
\mathcal{J} \in \operatorname{End}\left(T \oplus T^{*}\right)
$$

such that

$$
\mathcal{J}^{2}=-1
$$

and

$$
\mathcal{J}^{t} \mathcal{I} \mathcal{J}=\mathcal{I}
$$

\footnotetext{
${ }^{1} \mathrm{~A}$ similar situation arises in the projective superspace description of hyperkähler sigma models 4 .
} 
where $\mathcal{I}$ is the metric corresponding to the natural pairing of elements $X+\eta, Y+\lambda \in T \oplus T^{*}$ :

$$
<X+\eta, Y+\lambda>=\frac{1}{2}\left(\imath_{X} \lambda+\imath_{Y} \eta\right)
$$

The generalized almost complex structure is a (twisted) generalized complex structure iff it is integrable with respect to the (twisted) Courant bracket

$$
[X+\eta, Y+\lambda]_{C}:=[X, Y]_{L}+\mathcal{L}_{X} \lambda-\mathcal{L}_{Y} \eta-\frac{1}{2} d\left(\imath_{X} \lambda-\imath_{Y} \eta\right)+\imath_{X} \imath_{Y} H
$$

where $[X, Y]_{L}$ is the Lie-bracket and "twisted" refers to inclusion of the last term involving the closed three-form $H$. Integrability is defined by the requirement that the distributions defined by the projection operators

$$
\frac{1}{2}(1 \pm i \mathcal{J})
$$

are involutive with respect to the Courant-bracket. Of great importance to physical applications is the fact that the automorphisms of the Courant-bracket, in addition to diffeomorphisms, also contain the $b$-transform:

$$
e^{b}(X+\eta)=X+\eta+\imath_{X} b
$$

where $b$ is a closed two-form: $d b=0$. When acting on a generalized complex structure $\mathcal{J}$ it produces an equivalent generalized complex structure $\mathcal{J}_{b}$. In a matrix representation this reads;

$$
\mathcal{J}_{b}=\left(\begin{array}{ll}
1 & 0 \\
b & 1
\end{array}\right) \mathcal{J}\left(\begin{array}{cc}
1 & 0 \\
-b & 1
\end{array}\right)
$$

We now restrict our attention to a subset of generalized complex geometries: generalized Kähler geometries (GKG). These are GCG's with two commuting generalized complex structures $\mathcal{J}_{1,2}$ whose product defines a positive definite metric $\mathcal{G}$ on $T \oplus T^{*}$ that squares to the identity [1];

$$
\begin{aligned}
& {\left[\mathcal{J}_{1}, \mathcal{J}_{2}\right]=0} \\
& \mathcal{G}=-\mathcal{J}_{1} \mathcal{J}_{2} \\
& \mathcal{G}^{2}=1
\end{aligned}
$$

This is the proper setting for the bihermitean geometry of [3], which we now describe. 


\section{Bihermitean Geometry}

The data found in [3] to be necessary and sufficient to describe the target space geometry of a supersymmetric nonlinear sigma model with $(2,2)$ supersymmetry consists of two (ordinary) complex structures $J_{+}$and $J_{-}$, a metric $g$, hermitean with respect to both complex structures, and a closed three-form $H$ with a (local) two-form potential $B$.

The structures $\left(J_{ \pm}, g, B\right)$ satisfy

$$
\begin{aligned}
& J_{ \pm}^{2}=-1 \\
& J_{ \pm}^{t} g J_{ \pm}=g \\
& \nabla^{( \pm)} J_{ \pm}=0 \\
& H=d_{+}^{c} \omega_{+}=-d_{-}^{c} \omega_{-}
\end{aligned}
$$

where $\omega_{ \pm}$are the two-forms $g J_{ \pm}, d_{ \pm}^{c}$ is $d^{c}$ with respect to $J_{ \pm}$respectively, and the covariant derivatives have (Bismut) torsion [9]

$$
\nabla^{( \pm)}:=\nabla \pm g^{-1} H, \quad d H=0
$$

where $\nabla$ is the Levi-Civita connection.

When $J_{ \pm}$commute, it was found in [3] that there exist coordinates $z, \bar{z}$ and $z^{\prime}, \bar{z}^{\prime}$ that coordinatize $\operatorname{ker}\left(J_{+} \mp J_{-}\right)$, respectively, and for which the metric and three-form are given as derivatives of a function $K\left(z, \bar{z}, z, \bar{z}^{\prime}\right)$. Explicitly:

$$
\begin{aligned}
\omega_{ \pm}=\left(d_{\mp}^{c} d+d_{\Pi}^{c} d_{ \pm}^{c}\right) K \Leftrightarrow & g_{z \bar{z}}=\partial_{z} \partial_{\bar{z}} K, \quad g_{z^{\prime} \bar{z}^{\prime}}=-\partial_{z^{\prime}} \partial_{\bar{z}^{\prime}} K, \\
H=d d_{+}^{c} d_{-}^{c} K \Leftrightarrow & H_{z, \bar{z}, z^{\prime}}=\partial_{z} \partial_{\bar{z}} \partial_{z^{\prime}} K, \quad H_{z, \bar{z}, \bar{z}^{\prime}}=-\partial_{z} \partial_{\bar{z}} \partial_{\bar{z}^{\prime}} K, \\
& H_{z^{\prime}, \bar{z}^{\prime}, z}=-\partial_{z}^{\prime} \partial_{\bar{z}^{\prime}} \partial_{z} K, \quad H_{z^{\prime}, \bar{z}^{\prime}, \bar{z}}=\partial_{z^{\prime}} \partial_{\bar{z}^{\prime}} \partial_{\bar{z}} K
\end{aligned}
$$

where $d_{\Pi}^{c}$ is the $d^{c}$ operator defined with respect to the product $\Pi:=J_{+} J_{-}$and the differentials $d_{+}^{c}, d_{-}^{c}, d_{\Pi}^{c}$ anticommute.

A complete description for geometries with a nonvanishing coker $\left[J_{+}, J_{-}\right]$was an open problem until our paper [5]. Before describing the solution to the problem in sections 5 and 6. we recall Gualtieri's map of the bihermitean data $\left(J_{ \pm}, g, B\right)$ into Generalized Kähler Geometry [1]:

$$
\mathcal{J}_{1,2}=\left(\begin{array}{cc}
1 & 0 \\
B & 1
\end{array}\right)\left(\begin{array}{cc}
J_{+} \pm J_{-} & -\left(\omega_{+}^{-1} \mp \omega_{-}^{-1}\right) \\
\omega_{+} \mp \omega_{-} & -\left(J_{+}^{t} \pm J_{-}^{t}\right)
\end{array}\right)\left(\begin{array}{cc}
1 & 0 \\
-B & 1
\end{array}\right)
$$




$$
\mathcal{G}=\left(\begin{array}{ll}
1 & 0 \\
B & 1
\end{array}\right)\left(\begin{array}{cc}
0 & g^{-1} \\
g & 0
\end{array}\right)\left(\begin{array}{cc}
1 & 0 \\
-B & 1
\end{array}\right)
$$

Note that what looks like a $b$-transform is not, since $d B=H \neq 0$.

It is an interesting fact that if we introduce the usual GCS's corresponding to $J_{ \pm}$;

$$
\mathcal{J}_{ \pm}=\left(\begin{array}{cc}
J_{ \pm} & 0 \\
0 & -J_{ \pm}^{t}
\end{array}\right)
$$

the map (3.13) may be summarized as 2

$$
\mathcal{J}_{1,2}=\Pi_{+} \mathcal{J}_{+B} \pm \Pi_{-} \mathcal{J}_{-B},
$$

with

$$
\Pi_{ \pm}:=\frac{1}{2}(1 \pm \mathcal{G})
$$

\section{Poisson-structures}

There are three Poisson-structures relevant to our discussion. We first consider two real ones:

$$
\pi_{ \pm}:=\left(J_{+} \pm J_{-}\right) g^{-1}=-g^{-1}\left(J_{+} \pm J_{-}\right)^{t} .
$$

These were introduced in [10, where they were used essentially as described below, simplifying an earlier derivation in [11]. They ensure the existence of coordinates adapted to $\operatorname{ker}\left(J_{+}-J_{-}\right) \oplus \operatorname{ker}\left(J_{+}+J_{-}\right)$.

In a neighborhood of a regular point $x_{0}$ of $\pi_{-}$we may choose coordinates $x^{A}$ whose tangents lie in the kernel of $\pi_{-}$:

$$
\pi_{-}^{A \mu}=0, \Rightarrow J_{+\mu}^{A}=J_{-\mu}^{A} .
$$

Similarily, in a neighborhood of a regular point of $\pi_{+}$we have coordinates $x^{A^{\prime}}$ such that

$$
\pi_{+}^{A^{\prime} \mu}=0, \Rightarrow J_{+\mu}^{A^{\prime}}=-J_{-\mu}^{A^{\prime}}
$$

It then follows from the nondegeneracy of $\pi_{+} \pm \pi_{-}$that the Poisson brackets defined by $\pi_{+}$and by $\pi_{-}$cannot have common Casimir functions. In other words, the directions $A$ and $A^{\prime}$ cannot coincide. The result is that we may write

$$
J_{ \pm}=\left(\begin{array}{cccc}
* & * & * & * \\
* & * & * & * \\
0 & 0 & I_{c} & 0 \\
0 & 0 & 0 & \pm I_{t}
\end{array}\right)
$$

\footnotetext{
${ }^{2}$ This structure is related to how the map is derived in chapter 6 of [1]
} 
in coordinates adapted to

$$
\operatorname{ker}\left(J_{+}-J_{-}\right) \oplus \operatorname{ker}\left(J_{+}+J_{-}\right) \oplus \operatorname{coker}\left[J_{+}, J_{-}\right]
$$

where we write a canonical complex structure as

$$
I=\left(\begin{array}{cc}
i & 0 \\
0 & -i
\end{array}\right)
$$

and $c$ labels the $A$ and $t$ labels the $A^{\prime}$ directions.

A third Poisson structure $\sigma$, related to the real Poisson structures, was introduced in [12.

$$
\sigma:=\left[J_{+}, J_{-}\right] g^{-1}= \pm\left(J_{+} \mp J_{-}\right) \pi_{ \pm}=\mp\left(J_{+} \pm J_{-}\right) \pi_{\mp} .
$$

The relation to $\pi_{ \pm}$implies that

$$
\operatorname{ker} \sigma=\operatorname{ker} \pi_{+} \oplus \operatorname{ker} \pi_{-} \text {. }
$$

Tangents to the symplectic leaf for $\sigma$ lie in coker $\left[J_{+}, J_{-}\right]$. Using (4.25), we can use $\sigma$ to investigate the remaining directions in (4.21). We need that

$$
J_{ \pm} \sigma J_{ \pm}^{t}=-\sigma \Rightarrow \sigma=\sigma^{(2,0)}+\bar{\sigma}^{(0,2)}
$$

with the decomposition with respect to both $J_{+}$and $J_{-}$. Further $\sigma^{(2,0)}$ is holomorphic [12]:

$$
\bar{\partial} \sigma^{(2,0)}=0
$$

We now investigate the structure of the cokernel.

\section{The structure of coker $\left[J_{+}, J_{-}\right]$}

It is convenient to first treat the case $\operatorname{ker}\left[J_{+}, J_{-}\right]=\emptyset$. Then we have at our disposal a symplectic form $\Omega$ :

$$
\Omega:=\sigma^{-1}, \quad d \Omega=0, \quad J_{ \pm}^{t} \Omega J_{ \pm}=-\Omega .
$$

Choosing complex coordinates with respect to $J_{+}$,

$$
J_{+}=\left(\begin{array}{cc}
I_{s} & 0 \\
0 & I_{s}
\end{array}\right),
$$

with $I$ as in (4.23), we have the decomposition

$$
\Omega=\Omega_{+}^{(2,0)}+\bar{\Omega}_{+}^{(0,2)} .
$$


and

$$
\partial \Omega_{+}^{(2,0)}=0, \quad \bar{\partial} \Omega_{+}^{(2,0)}=0,
$$

which means in particular that $\Omega_{+}^{(2,0)}$ is holomorphic. We may then choose Darboux coordinates such that

$$
\Omega_{+}^{(2,0)}=d q^{a} \wedge d p^{a}, \quad \bar{\Omega}_{+}^{(0,2)}=d \bar{q}^{\bar{a}} \wedge d \bar{p}^{\bar{a}}
$$

Alternatively, we can choose complex coordinates with respect to $J_{-}$and similarily derive:

$$
\Omega_{-}^{(2,0)}=d Q^{a^{\prime}} \wedge d P^{a^{\prime}}, \quad \bar{\Omega}_{-}^{(0,2)}=d \bar{Q}^{\bar{a}^{\prime}} \wedge d \bar{P}^{\bar{a}^{\prime}}
$$

The transformation $\{q, p\} \rightarrow\{Q, P\}$ is a canonical transformations (symplectomorphism) and can thus be specified by a generating function $3(q, P)$. Thus in a neighborhood, the canonical transformation is given by the generating function $K(q, P)$

$$
p=\frac{\partial K}{\partial q}, \quad Q=\frac{\partial K}{\partial P} .
$$

We calculate all our geometic structures $J_{+}, J_{-}, \Omega$ and $g$ in the "mixed" coordinates $\{q, P\}$, using the transformation matrices

$$
\frac{\partial(q, p)}{\partial(q, P)}=\left(\begin{array}{cc}
1 & 0 \\
\frac{\partial p}{\partial q} & \frac{\partial p}{\partial P}
\end{array}\right)=\left(\begin{array}{cc}
1 & 0 \\
\frac{\partial^{2} K}{\partial q \partial q} & \frac{\partial^{2} K}{\partial P \partial q}
\end{array}\right):=\left(\begin{array}{cc}
1 & 0 \\
K_{L L} & K_{L R}
\end{array}\right)
$$

and

$$
\frac{\partial(Q, P)}{\partial(q, P)}=\left(\begin{array}{cc}
\frac{\partial Q}{\partial q} & \frac{\partial Q}{\partial P} \\
0 & 1
\end{array}\right)=\left(\begin{array}{cc}
\frac{\partial^{2} K}{\partial q \partial P} & \frac{\partial^{2} K}{\partial P \partial P} \\
0 & 1
\end{array}\right):=\left(\begin{array}{cc}
K_{R L} & K_{R R} \\
0 & 1
\end{array}\right)
$$

where the labels $L$ and $R$ are shorthand for the coordinates $\left\{q^{a}, \bar{q}^{\bar{a}}\right\}$ and $\left\{P^{a^{\prime}}, \bar{P}^{\bar{a}^{\prime}}\right\}$ in (5.32) and (5.33) above. The expressions for $J_{ \pm}$are nonlinear and can be read off from the formulae given below for the general case with $\operatorname{ker}\left[J_{+}, J_{-}\right] \neq \emptyset$. The symplectic structure $\Omega$ is the only linear function of $K$

$$
\Omega=K_{A A^{\prime}} d q^{A} \wedge d P^{A^{\prime}}
$$

where we use the collective notation $A=\{a, \bar{a}\}, A^{\prime}=\left\{a^{\prime}, \bar{a}^{\prime}\right\}$. We write $\Omega$ as a matrix

$$
\Omega=\left(\begin{array}{cc}
0 & K_{L R} \\
-K_{R L} & 0
\end{array}\right)
$$

and find the metric and $B$-field using this matrix as follows [14] (cf. 4.24)

$$
g=\Omega\left[J_{+}, J_{-}\right], \quad B=\Omega\left\{J_{+}, J_{-}\right\} .
$$

\footnotetext{
${ }^{3}$ There always exists at least one polarization such that any symplectomorphism, can be written in terms of such a generating function 13 .
} 


\section{The general case}

In the general case when both $\operatorname{ker}\left[J_{+}, J_{-}\right]$and coker $\left[J_{+}, J_{-}\right]$are nonempty, we combine the discussion leading to (4.21) in section 4 with that of the previous section. The formulas that we compute are rather complicated, and we have not found a suitable coordinate free way to express them.

We assume that in a neighborhood of $x_{0}$, the ranks of $\pi_{ \pm}$are constant, and as result, the rank of $\sigma$ is constant. We work in coordinates $\left\{q, p, z, z^{\prime}\right\}$ adapted to the symplectic foliation of $\sigma$ as well as to the description of $\operatorname{ker}\left[J_{+}, J_{-}\right]$given in section 4. In such coordinates $J_{+}$may be taken to have the canonical form 4

$$
J_{+}=\left(\begin{array}{cccc}
I_{s} & 0 & 0 & 0 \\
0 & I_{s} & 0 & 0 \\
0 & 0 & I_{c} & 0 \\
0 & 0 & 0 & I_{t}
\end{array}\right)
$$

where $\{q, p\}$ are Darboux coordinates for a symplectic leaf of $\sigma$ and $\left\{z, z^{\prime}\right\}$ parametrize the kernels of $\pi_{\mp}$. Similarily, there are coordinates $\left\{Q, P, z, z^{\prime}\right\}$ where $J_{-}$takes a diagonal form (identical to that of $J_{+}$in (6.40) except for a change of sign in the last entry; $I_{t} \rightarrow-I_{t}$ ). For every leaf separately we may now apply the arguments of section 5 . There thus exists a generating function $K\left(q, P, z, z^{\prime}\right)$ for the symplectomorphisms between the two sets of coordinates. The transformation matrices to the coordinates $\left\{q, P, z, z^{\prime}\right\}$ are given by the obvious extensions of (5.35) and (5.36) to include $z, z^{\prime}$. The expression for $J_{ \pm}$in the "mixed" coordinates are thens:

$$
J_{+}=\left(\begin{array}{cccc}
I_{s} & 0 & 0 & 0 \\
K_{R L}^{-1} C_{L L} & K_{R L}^{-1} I_{s} K_{L R} & K_{R L}^{-1} C_{L c} & K_{R L}^{-1} C_{L t} \\
0 & 0 & I_{c} & 0 \\
0 & 0 & 0 & I_{t}
\end{array}\right),
$$

and

$$
J_{-}=\left(\begin{array}{cccc}
K_{L R}^{-1} I_{s} K_{R L} & K_{L R}^{-1} C_{R R} & -K_{L R}^{-1} C_{R c} & K_{L R}^{-1} A_{R t} \\
0 & -I_{s} & 0 & 0 \\
0 & 0 & I_{c} & 0 \\
0 & 0 & 0 & -I_{t}
\end{array}\right)
$$

where

$$
K_{L R}^{-1}=\left(K_{R L}\right)^{-1}
$$

\footnotetext{
${ }^{4}$ For historical reasons related to the origin in sigma models involving chiral and twisted chiral superfields, we again use the labels $c$ and $t$ for the $z$ and $z^{\prime}$ directions.

${ }^{5}$ The rows and columns of this matrix correspond to the directions along $\left\{q, P, z, z^{\prime}\right\}$.
} 


$$
\begin{aligned}
& C=I K-K I=\left(\begin{array}{cc}
0 & 2 i K \\
-2 i K & 0
\end{array}\right), \\
& A=I K+K I=\left(\begin{array}{cc}
2 i K & 0 \\
0 & -2 i K
\end{array}\right),
\end{aligned}
$$

where we suppress the indices in the last two entries6. The relations (5.39) no-longer hold when $\operatorname{ker}\left[J_{+}, J_{-}\right] \neq \emptyset$. The definition (4.24) of the Poisson structure $\sigma$ still determines the metric, except along the kernel. However, the additional relation $H= \pm d_{ \pm}^{c} \omega_{ \pm}$from (3.10) provides us with an equation for the remaining components of $g$ and allows us to find the $B$-field. From the sigma model we already know the solution; the sum $E=\frac{1}{2}(g+B)$ of the metric $g$ and $B$-field takes on the explicit form:

$$
\begin{aligned}
E_{L L} & =C_{L L} K_{L R}^{-1} I_{s} K_{R L} \\
E_{L R} & =I_{s} K_{L R} I_{s}+C_{L L} K_{L R}^{-1} C_{R R} \\
E_{L c} & =K_{L c}+I_{s} K_{L c} I_{c}+C_{L L} K_{L R}^{-1} C_{R c} \\
E_{L t} & =-K_{L t}-I_{s} K_{L t} I_{t}+C_{L L} K_{L R}^{-1} A_{R t} \\
E_{R L} & =-K_{R L} I_{s} K_{L R}^{-1} I_{s} K_{R L} \\
E_{R R} & =-K_{R L} I_{s} K_{L R}^{-1} C_{R R} \\
E_{R c} & =K_{R c}-K_{R L} I_{s} K_{L R}^{-1} C_{R c} \\
E_{R t} & =-K_{R t}-K_{R L} I_{s} K_{L R}^{-1} A_{R t} \\
E_{c L} & =C_{c L} K_{L R}^{-1} I_{s} K_{R L} \\
E_{c R} & =I_{c} K_{c R} I_{s}+C_{c L} K_{L R}^{-1} C_{R R} \\
E_{c c} & =K_{c c}+I_{c} K_{c c} I_{c}+C_{c L} K_{L R}^{-1} C_{R c} \\
E_{c t} & =-K_{c t}-I_{c} K_{c t} I_{t}+C_{c L} K_{L R}^{-1} A_{R t} \\
E_{t L} & =C_{t L} K_{L R}^{-1} I_{s} K_{R L} \\
E_{t R} & =I_{t} K_{t R} I_{s}+C_{t L} K_{L R}^{-1} C_{R R} \\
E_{t c} & =K_{t c}+I_{t} K_{t c} I_{c}+C_{t L} K_{L R}^{-1} C_{R c} \\
E_{t t} & =-K_{t t}-I_{t} K_{t t} I_{t}+C_{t L} K_{L R}^{-1} A_{R t}
\end{aligned}
$$

In view of this last relation (6.44) as well as (6.41), (6.42) and (5.38) it seems appropriate to call $K$ the generalized Kähler potential.

\footnotetext{
${ }^{6}$ In (6.43), the rows and columns correspond to $\{A, \bar{A}\}$, where $\{A\}$ are any one of the $\left\{q, P, z, z^{\prime}\right\}$ directions in (6.41), (6.42), (6.44).
} 


\section{Linearization of Generalized Kähler geometry}

As can be seen in (6.44), the expressions for the metric and the $B$-field in terms of the generalized Kähler potential are highly nonlinear in contrast to what is the case in ordinary Kähler geometry. The nonlinearities all stem from the cokernel of $\left[J_{+}, J_{-}\right]$: when $\left[J_{+}, J_{-}\right]=$ 0 , the metric and B-field can be expressed as linear functions of the Hessian of $K$. We note that the structure of the nonlinearities are such as one might expect from a quotient construction. One is therefore faced with a natural question: Is there a space in which all the geometric data is encoded linearly with respect to the generalized Kähler potential and which gives the nonlinear Generalized Kähler geometry through a quotient construction?

Inspired by superspace sigma-models, we have found the following local prescription for generating such a space: In local coordinates, we simply make the substitution

$$
q \rightarrow z_{L}+z_{L}^{\prime}, \quad P \rightarrow z_{R}+\bar{z}_{R}^{\prime}
$$

in the Generalized Kähler potential $K$ and use the naive commuting complex structures $J_{ \pm}$ in which the $z$-coordinates are biholomorphic and the $z^{\prime}$-coordinates are $J_{+}$holomorphic and $J_{-}$antiholomorphic. This introduces isometries of the metric (3.12) corresponding to shifts of $z_{L}, z_{L}^{\prime}$ that preserve the sum, and similarly for $z_{R}, \bar{z}_{R}$; taking a quotient with respect to these isometries leads to the original nonlinear model. We now attempt to make mathematical sense of this prescription. We begin with some definitions:

Definition 1 (BiLP). A Generalized Kähler Geometry with commuting complex structures will be called a Bihermitean Local Product space (BiLP).

We also give a special name to a BiLP with the isometries sketched above:

Definition 2 (ALP). A BiLP with these additional isometries (further characterized below) we will call an Auxilliary Product Space (ALP).

The isometries are generated by $2 n$ complex Abelian Killing vectors that preserve all the BiLP geometric data. They can be separated into two groups of $n$ Killing vectors $\left\{k_{L A}\right\}$ and $\left\{k_{R A}\right\}, A=1 \ldots n$, satisfying the further requirement

$$
\imath_{k_{L A}} H=d \alpha_{L A}, \quad \imath_{k_{R A^{\prime}}} H=-d \alpha_{R A^{\prime}},
$$

where the one-form $\alpha$ is the dual of the killing vector $k$ : $\alpha=g(k)$; this is equivalent to saying that the Killing vectors are covariantly constant with respect to the covariant derivative with torsion (3.11). Such Killing vectors are called Kac-Moody Killing vectors. 
We require that each group of Killing vectors $k_{L}$ and $k_{R}$ by themselves span maximally isotropic subspaces, i.e., that

$$
g\left(k_{L A}, k_{L B}\right)=0, \quad g\left(k_{R A^{\prime}}, k_{R B^{\prime}}\right)=0,
$$

but that the inner product

$$
h_{A^{\prime} B}=g\left(k_{R A^{\prime}}, k_{L B}\right)
$$

is nondegenerate.

Theorem. Locally, any generalized Kähler manifold $M$ is a quotient of an ALP by its Kac-Moody isometries.

The quotient is performed by going to the orbits of the action of the Killing vector and choosing a horizontal subspace by specifying a connection. In the particular case with left and right Kac-Moody Killing vectors there is a corresponding left and right connection given by

$$
\left(\theta_{R}\right)_{\mu}^{A^{\prime}}=g_{\mu \nu} k_{L B}^{\nu} h^{B A^{\prime}}, \quad\left(\theta_{L}\right)_{\mu}^{A}=h^{A B^{\prime}} k_{R B^{\prime}}^{\nu} g_{\nu \mu},
$$

where $h^{A B^{\prime}}$ is the inverse of the nondegenerate matrix (7.48). The connections (7.49) satisfy the following properties

$$
\theta_{L}\left(k_{L}\right)=\mathbf{1}, \quad \theta_{R}\left(k_{R}\right)=\mathbf{1}, \quad \theta_{L}\left(k_{R}\right)=0, \quad \theta_{R}\left(k_{L}\right)=0 .
$$

Vectors in the horizontal subspaces are defined as the vectors lying in the kernel of both $\theta_{L}$ and $\theta_{R}$.

All geometric structures defined on the ALP can now be defined on the quotient space. As usual, forms are projected using the connection so that the contraction with any vertical vector is zero, whereas vectors are pushed forward to the quotient using the bundle projection p. When we project the complex structures

$$
\tilde{J}_{ \pm}(X):=\mathbf{p}_{*}\left(J_{ \pm}(X)-\theta_{L}(X) \cdot J_{ \pm}\left(k_{L}\right)-\theta_{R}(X) \cdot J_{ \pm}\left(k_{R}\right)\right) \forall X
$$

it is clear that the projected complex structures $\tilde{J}_{ \pm}$do not commute even though $J_{ \pm}$do.

Projecting the metric and $B$-field, we find

$$
\begin{aligned}
\tilde{g} & =g-h\left(\theta_{L}, \theta_{R}\right)-h\left(\theta_{R}, \theta_{L}\right), \\
\tilde{B} & =B-h\left(\theta_{L}, \theta_{R}\right)+h\left(\theta_{R}, \theta_{L}\right) .
\end{aligned}
$$

It is straightforward to see that $\tilde{g}$ gives zero when contracted with any vertical vector while this is not so for $\tilde{B}$. This (apparent) mystery is resolved by showing that $\tilde{H}=d \tilde{B}$ can be written as

$$
\tilde{H}=H-\theta_{L} \wedge \imath_{k_{L}} H-\theta_{R} \wedge \imath_{k_{R}} H-\theta_{L} \wedge \theta_{R} \wedge \imath_{k_{L}} \imath_{k_{R}} H
$$


so that the geometrically meaningful object $\tilde{H}$ defined by $d \tilde{B}$ is indeed well defined on the quotient. The quotients $\tilde{g}$ and $\tilde{H}$ agree with the expressions for the original generalized Kähler manifold.

\section{Acknowledgement:}

We are grateful to the 2006 Simons Workshop for providing the stimulating atmosphere where this work was initiated. The work of UL was supported by EU grant (Superstring theory) MRTN-2004-512194 and VR grant 621-2003-3454. The work of MR was supported in part by NSF grant no. PHY-0354776. The research of R.v.U. was supported by Czech ministry of education contract No. MSM0021622409. The research of M.Z. was supported by VR-grant 621-2004-3177

\section{References}

[1] M. Gualtieri, "Generalized complex geometry," Oxford University DPhil thesis, arXiv:math.DG/0401221.

[2] N. Hitchin, "Generalized Calabi-Yau manifolds," Q. J. Math. 54 (2003), no. 3, 281 308, arXiv:math.DG/0209099.

[3] S. J. Gates, C. M. Hull and M. Roček, "Twisted Multiplets And New Supersymmetric Nonlinear Sigma Models," Nucl. Phys. B248 (1984) 157.

[4] U. Lindström and M. Roček, private communication, in preparation.

[5] U. Lindström, M. Rocek, R. von Unge and M. Zabzine, "Generalized Kaehler manifolds and off-shell supersymmetry," Commun. Math. Phys. 269, 833 (2007) arXiv:hep-th/0512164.

[6] U. Lindström, M. Roček, R. von Unge and M. Zabzine, "Linearizing generalized Kaehler geometry," arXiv:hep-th/0702126.

[7] U. Lindström, R. Minasian, A. Tomasiello and M. Zabzine, "Generalized complex manifolds and supersymmetry," Commun. Math. Phys. 257, 235 (2005) arXiv:hep-th/0405085.

[8] M. Zabzine, "Lectures on generalized complex geometry and supersymmetry," arXiv:hep-th/0605148. 
[9] K. Yano, "Differential geometry on complex and almost complex spaces," (Pergamon, Oxford, 1965)

[10] S. Lyakhovich and M. Zabzine, "Poisson geometry of sigma models with extended supersymmetry," Phys. Lett. B548 (2002) 243 arXiv:hep-th/0210043].

[11] I. T. Ivanov, B. B. Kim and M. Roček, "Complex structures, duality and WZW models in extended superspace," Phys. Lett. B343 (1995) 133 arXiv:hep-th/9406063.

[12] N. Hitchin, "Instantons, Poisson structures and generalized Kähler geometry," arXiv:math.DG/0503432.

[13] V. I. Arnold, "Mathematical methods of classical mechanics," Translated from the Russian by K. Vogtmann and A. Weinstein. Second edition. Graduate Texts in Mathematics, 60. Springer-Verlag, New York, 1989. xvi+508 pp.

[14] J. Bogaerts, A. Sevrin, S. van der Loo and S. Van Gils, "Properties of semichiral superfields," Nucl. Phys. B562 (1999) 277 arXiv:hep-th/9905141].

[15] T. Buscher, U. Lindström and M. Roček, "New Supersymmetric Sigma Models With Wess-Zumino Terms," Phys. Lett. B202, 94 (1988). 